\title{
ACCURATE OPTICAL POSITIONS USING THE PALOMAR
}

\author{
SKY ATLAS
}

\author{
R. W. HUNSTEAD
}

Cornell-Sydney University Astronomy Centre, School of Physics, University of Sydney, Sydney, Australia

\begin{abstract}
Optical positions measured directly on the prints and plates of the Palomar Sky Atlas have been compared with various published lists of precise optical positions. The external comparisons suggest that positional errors $\sim \pm 0$ ". 3 in each coordinate have been achieved. This supports the claimed accuracy and establishes the Sky Atlas as an important astrometric medium for investigating radio-optical associations.
\end{abstract}

\section{Introduction}

The measurement of precise optical positions for the objects associated with radio sources presents some special astrometric problems. The most important of these results from the shape of the radio luminosity function: the strongest radio sources tend also to be the faintest optically, being mostly fainter than $17^{m}$. This effectively rules out the conventional astrograph for routine identification and astrometric work. The Sky Atlas prepared from the Palomar Observatory Sky Survey (POSS) has therefore been used almost exclusively as the initial search medium for optical identifications, because it reaches faint magnitudes $\left(20^{m}-21^{m}\right)$ and also offers colour information. However, the view has been widely held that the Sky Atlas prints are quite unsuitable for measuring positions to better than a few arc seconds due to distortions of the paper base (Dewhirst, 1963). Consequently, the much-needed astrometry continues to be carried out on Schmidt plates specially taken for this purpose at Palomar, Cambridge and Asiago.

The aim of the present paper is to examine in detail the accuracy of direct measurements on the second-generation copies of the POSS in relation to published lists of optical positions obtained from original plate material. The POSS position measurements upon which the following analysis is based have been reported in an earlier paper (Hunstead, 1971), together with a description of the measuring engine and the measurement and reduction techniques.

\section{Comparisons with Other Optical Positions}

The most general method of assessing the external accuracy of different sets of measurements is to evaluate unweighted variances about a mean difference for each comparison. Systematic differences will be influenced primarily by regional fluctuations amongst the different star catalogues and a discussion of these effects will be deferred until a later paper.

The six lists of optical positions used in the present comparison are given below with the reference star catalogues used. 


\begin{tabular}{|c|c|c|}
\hline Observers & Abbreviation & Catalogue \\
\hline (i) Argue and Kenworthy (1972) & & AGK3 \\
\hline Argue et al. (1973) & AK & $\mathrm{AGK} 3, \mathrm{SAO}$ \\
\hline (ii) Barbieri et al. (1971) & & SAO \\
\hline Barbieri et al. (1972) & BLGP & AGK3, SAO \\
\hline (iii) Bolton (1968) & B & Yale \\
\hline (iv) Hunstead (1971) & $\mathrm{H}$ & $\mathrm{SAO}$ \\
\hline (v) Kristian and Sandage (1970) & $\mathrm{KS}$ & $\mathrm{AGK} 3, \mathrm{SAO}$ \\
\hline $\begin{array}{l}\text { (vi) Véron (1956a, b, 1966, 1968); } \\
\text { Bolton et al. (1965); Sandage et al. (1965) }\end{array}$ & V & AGK2, SAO \\
\hline
\end{tabular}

With the exception of (iii) and (iv), the positions were mostly referred to AGK north of $-2^{\circ}$ and SAO southwards.

The results of inter-comparisons amongst sources common to each pair of lists are summarised in Table I, which gives the (unweighted) standard deviation $\sigma^{\prime}$ about a mean difference for each comparison $\left(\sigma^{\prime}=\frac{1}{2}\left(\sigma_{\alpha}+\sigma_{\delta}\right)\right)$. The diagonal entries in the table correspond to the external accuracies claimed for each list.

\section{TABLE I}

Standard deviations (mean of R.A. and Decl.) in a single position difference amongst various sets of optical position measurements. The underlined entries along the diagonal give the external errors estimated by each observer. A dash $(-)$ indicates fewer than five objects in common.

\begin{tabular}{lllllll}
\hline & AK & BCGP & B & H & KS & V \\
\hline AK & 0.2 & 0.68 & - & 0.34 & 0.30 & 1.0 \\
BCGP & & $\underline{0 \prime 4}$ & 1.2 & 0.75 & 0.70 & 1.3 \\
B & & & $\underline{0.5}$ & 1.1 & 1.3 & - \\
H & & & & $\underline{0.4}$ & 0.38 & 1.1 \\
KS & & & & & $\underline{0.3}$ & 1.0 \\
V & & & & & & $\underline{1.0}$ \\
\hline
\end{tabular}

Despite the fact that a different number (always $\geqslant 6$ ) and selection of sources were used in each comparison, the data in Table I give a surprisingly consistent picture of the intrinsic accuracy of each list of positions. Only in the cases of B and BCGP do the actual errors appear to be underestimated. Most important, however, are the comparisons amongst $\mathrm{AK}, \mathrm{KS}$ and $\mathrm{H}$ which suggest that the direct measurements on the POSS are statistically accurate at the 0.3 level. It is worthwhile placing the information of Table I in better perspective by considering the intrinsic accuracy $(\sigma)$ of each list in terms of the observer effort. We may regard $1 / \sigma$ as measuring the 'return' on an 'investment' of $N$ measurements, where $N$ is defined here as the mean number of separate $\mathbf{x}, \mathbf{y}$ measurements for a concluded position. The quantity $E=100 / \sigma N$ therefore provides an 'efficiency measure' for each list of positions. Figure 1 shows $\sigma$ plotted as a function of $N$, with lines corresponding to isophotes of $E$.

The information in Figure 1 may be interpreted in several ways. From the viewpoint 
of efficiency and convenience alone, the present technique using the Sky Survey is clearly superior. However, there are also situations that appear to demand the most precise optical positions, such as,

(a) defining zero-point corrections for precise radio measurements when the number of calibrators is small (Smith, 1971); and

(b) the more general problem of investigating radio-optical displacements for the most compact radio sources.

The former problem can obviously be overcome by choosing a larger number of calibrators, although this may not always be practicable for radio telescopes with

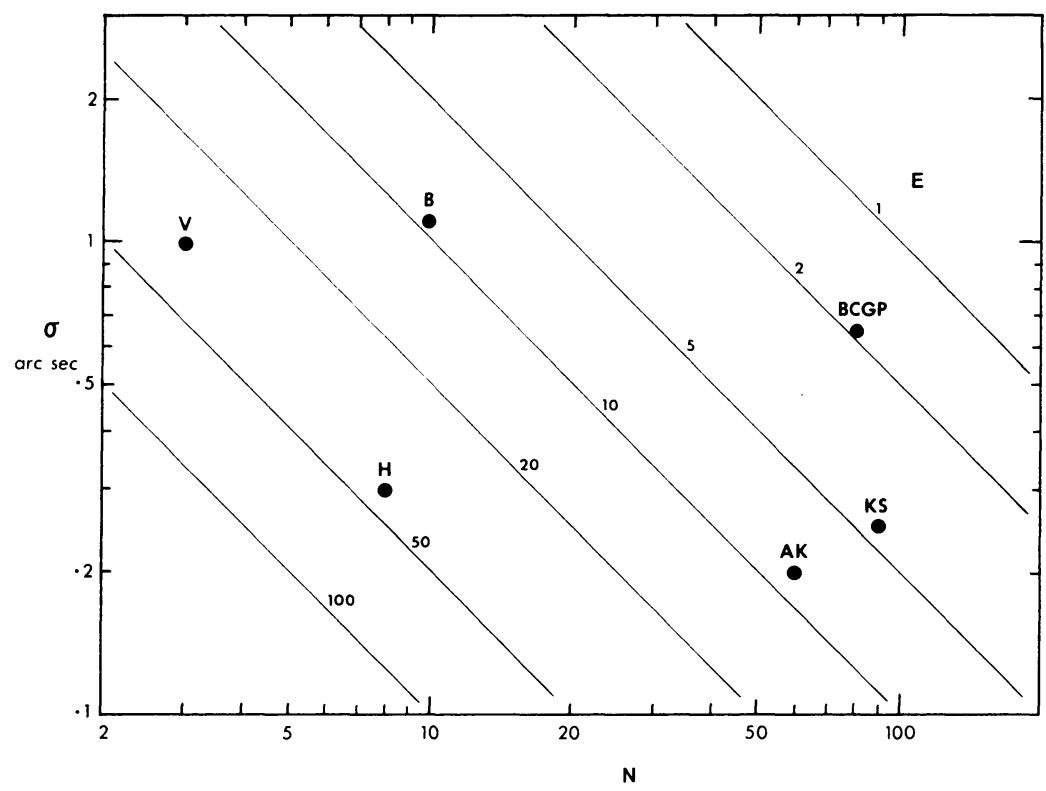

Fig. 1. Plot of the estimated external error, $\sigma$ (arc seconds), against the number, $N$, of separate $x, y$ measurements for a concluded position in each of the six optical lists. The lines correspond to isophotes of 'efficiency measure', $E$, where $E=100 / \sigma N$.

beams of the order of a few arc seconds; the problem should nevertheless be only a temporary one. The degree to which the second problem can be pursued will probably be determined primarily by the limitations of conventional photographic astrometry, i.e. measurement errors, plate distortions, photographic noise and 'warps' in the fundamental star catalogues. At present it would seem to be more profitable astrophysically to direct the optical effort towards an investigation of spatial relationships between optical sources and radio structures on the scale of a few arc seconds. For this purpose, astrometry using the Sky Atlas would permit an extensive quantitative study of the physics of non-thermal sources, as well as enabling reliable identifications to be made at a rate far greater than is feasible with specially taken plates. A further advantage is that this work may be undertaken by the radio astronomers themselves. 


\section{Outlook}

The question now arises as to whether it is possible to improve significantly on the 0 ". 3 figure derived earlier for the POSS measurements. The answer is almost certainly no - not without eroding the present advantage in efficiency. An analysis of repeated measurements gives an internal error of $0.27 \mathrm{rms}$ in each coordinate while the uncertainty of fit to SAO will be $\sim 0$. $3 / \sqrt{8}=0$. $11 \mathrm{rms}$; combining these gives an estimated external error of 0 .'29, in close agreement with the value of 0.30 deduced from the earlier comparisons. The 0 ".27 internal error can be attributed mainly to photographic noise at the QSO or galaxy level affecting the setting accuracy. This value is surprisingly small when account is taken of the variability in image quality from print to print and between the centre and edge of each print, quite apart from the overall deterioration resulting from the two additional photographic processes. The use of fine-grained IIIa-J emulsions for the southern sky survey to be commenced shortly by the British 48 -in. Schmidt telescope may be expected to give a reduction in this noise component.

A further point worth mentioning concerns the star catalogues presently available for the southern sky. A major portion of the positional errors in the SAO at epoch 1950 arise from propagated uncertainties in the stellar proper motions (Dieckvoss, 1963). The second-epoch plates for the Southern Yale Zone Catalogues, which contribute more than $50 \%$ of the SAO stars south of the equator, were taken in 1933 . Since most of the POSS was carried out between 1950 and 1955, it is clear that on average the random and systematic errors in an SAO reference frame defined on the POSS will be only $\frac{1}{2}-\frac{2}{3}$ as serious as those on recent plates using the same star field. This in turn means that an SAO reference frame specified by 8 stars with a favourable distribution on the POSS plates is more precisely defined than one specified by $\sim 20$ standards on a recent plate. The residuals quoted by Barbieri et al. (1972) tend to support this argument.

\section{Conclusions}

The principal advantage of the POSS lies in its demonstrably greater efficiency in securing unambiguous radio-optical associations. It is clear from the foregoing analysis that direct position measurements on the POSS give adequate precision for all but the most demanding situations. In the latter case, it is arguable whether a uniform, absolute accuracy of 0 . 1 or better can in fact be achieved with conventional optical astrometry to match the anticipated precision of radio astrometry.

\section{Acknowledgements}

This work has been supported by the Australian Research Grants Committee, The Sydney University Research Grants Committee, and the Science Foundation for Physics within the University of Sydney. 


\section{References}

Argue, A. N. and Kenworthy, C. M.: 1972, Monthly Notices Roy. Astron. Soc. 160, 197.

Argue, A. N., Kenworthy, C. M., and Stewart, P. M.: 1973, Astrophys. Letters 14, 99.

Barbieri, C., Capaccioli, M., and Pinto, G.: 1971, Contrib. Obs. Astrofis. Asiago, No. 245.

Barbieri, C., Capaccioli, M., Ganz, R., and Pinto, G.: 1972, Astron. J. 77, 444.

Bolton, J. G.: 1968, Publ. Astron. Soc. Pacific 80, 5.

Bolton, J. G., Clarke, M. E., Sandage, A. R., and Véron, P.: 1965, Astrophys. J. 142, 1289.

Dewhirst, D. W.: 1963, in H. P. Palmer, R. D. Davies, and M. I. Large (eds.), Radio Astronomy Today, Harvard University Press, p. 178.

Dieckvoss, W.: 1963, Stars and Stellar Systems 3, 40.

Hunstead, R. W.: 1971, Monthly Notices Roy. Astron. Soc. 152, 277.

Kristian, J. and Sandage, A.: 1970, Astrophys. J. 162, 391.

Sandage, A., Véron, P., and Wyndham, J. D.: 1965, Astrophys. J. 142, 1307.

Smith, J. W.: 1971, Nature Phys. Sci. 232, 150.

Véron, P.: 1965a, Astrophys. J. 141, 332.

Véron, P.: 1965b, Astrophys. J. 141, 1284.

Véron, P.: 1966, Astrophys. J. 144, 861.

Véron, P.: 1968, Ann. Astrophys. 31, 483.

\section{DISCUSSION}

Dieckvoss: It is better to include 3rd order distortion of $0.36 / 1^{\circ}$. At Bergedorf diapositives from sky survey charts are measured with the 2-screw Mann machine. Carte du Ciel charts may also be measured (to 14th limiting mag.).

Hunstead: The Schmidt bending correction is very small in comparison with the stretching effects on the Sky Survey prints. However, it is a systematic effect and maybe should be included.

Luyten: Our own experience agrees with what you have stated: we find that with our automatic measuring machine we get mean errors for a single image measurement on one plate of \pm 0 ". 15 from overlapping plates.

Eichhorn: I don't want to argue whether it is desirable to go to higher accuracy than that corresponding to 0.4 or 0.3 standard error, but more sophisticated astrometric methods can certainly achieve higher accuracy, although probably not on the Palomar Schmidt paper prints.

Hunstead: I was talking about objects generally fainter than 18 th mag.

Elsmore: In confirmation of Dr Hunstead's claims, I would like to report that at the Institute of Astronomy, Cambridge C. Hazard and A. N. Argue have made a similar investigation and find that very similar accuracies to those you report can be achieved, working from the prints.

Van Altena: How close are the reference stars to the radio source, and do you encounter much paper stretch?

Hunstead: The reference stars are always within $\sim 0.9$ degrees due to the limitations of the measuring engine.

We do find paper stretch but it appears to be very uniform from print to print.

Wall: With Dr Hunstead's kind co-operation, a version of the simple measuring engine in the Department of Astrophysics, Sydney University, was constructed at the Parkes Observatory. Subsequently, measurements of a number of optical positions of radio sources have been made from the Parkes copies of prints of the Palomar Sky Survey. Setting residuals are $\pm 2 \mu \mathrm{m}$, similar to those found by Dr. Hunstead for the Sydney University measurements, while the mean residuals for the reference stars (taken from the SAO catalogue) are $\sim 0$. 5 . These mean residuals are slightly larger than those found by Dr Hunstead; the difference may be due to the controlled environment in which the Sydney University prints are kept. 